\title{
Corrigendum: An In vitro Study of Bio-Control and Plant Growth Promotion Potential of Salicaceae Endophytes
}

\section{OPEN ACCESS}

\section{Approved by: \\ Frontiers in Microbiology Editorial \\ Office, \\ Frontiers Media SA, Switzerland \\ ${ }^{*}$ Correspondence: Sharon L. Doty sldoty@uw.edu}

Specialty section: This article was submitted to Microbial Symbioses, a section of the journal

Frontiers in Microbiology

Received: 09 May 2018 Accepted: 13 August 2018 Published: 05 September 2018

Citation:

Kandel SL, Firrincieli A, Joubert PM, Okubara PA, Leston ND, McGeorge KM, Mugnozza GS, Harfouche A, Kim S-H and Doty SL (2018) Corrigendum: An In vitro Study of Bio-Control and Plant Growth Promotion Potential of Salicaceae Endophytes. Front. Microbiol. 9:2067. doi: 10.3389/fmicb.2018.02067

\begin{abstract}
Shyam L. Kandel ${ }^{1}$, Andrea Firrincieli ${ }^{2}$, Pierre M. Joubert ${ }^{3}$, Patricia A. Okubara ${ }^{4}$, Natalie D. Leston ${ }^{5}$, Kendra M. McGeorge ${ }^{5}$, Giuseppe S. Mugnozza ${ }^{2}$, Antoine Harfouche ${ }^{2}$, Soo-Hyung $\mathrm{Kim}^{1}$ and Sharon L. Doty ${ }^{1 *}$

${ }^{1}$ School of Environmental and Forest Sciences, College of the Environment, University of Washington, Seattle, WA, United States, ${ }^{2}$ Department for Innovation in Biological, Agro-Food and Forest Systems, University of Tuscia, Viterbo, Italy, ${ }^{3}$ Department of Biology, University of Washington, Seattle, WA, United States, ${ }^{4}$ Wheat Health, Genetics and Quality Research Unit, USDA-ARS, Pullman, WA, United States, ${ }^{5}$ Department of Plant Pathology, Washington State University, Pullman, WA, United States
\end{abstract}

Keywords: bio-control, Salicaceae endophytes, soil borne plant pathogens, Burkholderia

\section{A Corrigendum on}

An In vitro Study of Bio-Control and Plant Growth Promotion Potential of Salicaceae Endophytes

by Kandel, S. L., Firrincieli, A., Joubert, P. M., Okubara, P. A., Leston, N. D., McGeorge, K. M., et al. (2017). Front. Microbiol. 8:386. doi: 10.3389/fmicb.2017.00386

In the original article, there was a mistake in Table $\mathbf{1}$ as published. The correct GenBank accession number of strains; WP 4-3-1, WP 4-4-2, WP 4-4-6, WP 4-5-3, and WP42 are KU500895, KU500894, KU500891, KU500892, and KF597276, respectively. The correct identity of strains WP 4-4-2 and WP 4-5-3 is Rahnella sp. The accession number of strains; WP 4-3-1, WP 4-4-2, WP 4-4-6, WP 4-5-3, and WP42 were misprinted. The identity of strains WP 4-4-2 and WP 4-5-3 was listed as Rahnella aquatilis. The corrected Table $\mathbf{1}$ appears below.

The authors apologize for this error and state that this does not change the scientific conclusions of the article in any way.

The original article has been updated.

Conflict of Interest Statement: The authors declare that the research was conducted in the absence of any commercial or financial relationships that could be construed as a potential conflict of interest.

Copyright () 2018 Kandel, Firrincieli, Joubert, Okubara, Leston, McGeorge, Mugnozza, Harfouche, Kim and Doty. This is an open-access article distributed under the terms of the Creative Commons Attribution License (CC BY). The use, distribution or reproduction in other forums is permitted, provided the original author(s) and the copyright owner(s) are credited and that the original publication in this journal is cited, in accordance with accepted academic practice. No use, distribution or reproduction is permitted which does not comply with these terms. 
TABLE 1 | Endophyte strains used in this experiment.

\begin{tabular}{|c|c|c|c|c|c|c|}
\hline Strain & Identity & Origin & Appearance & PGPA $^{a}$ & $\begin{array}{l}\text { GenBank } \\
\text { accession no. }\end{array}$ & $\begin{array}{l}\text { rRNA sequence } \\
\text { size, partial (bp) }\end{array}$ \\
\hline WP 4-2-2 & Burkholderia sp. & Poplar root & Buff & IAA, NA & KU495920 & 903 \\
\hline WP 4-3-2 & Burkholderia sp. & Poplar stem & Buff, mucoid & $\mathrm{Sd}, \mathrm{NA}$ & KU500893 & 947 \\
\hline WP 4-3-3 & Curtobacterium sp. & Poplar stem & Bright yellow & IAA & KU550576 & 962 \\
\hline WP 4-4-2 & Rahnella sp. & Poplar stem & Buff, mucoid & IAA, Sd & KU500894 & 966 \\
\hline WP 4-5-3 & Rahnella sp. & Poplar stem & Buff, mucoid & Sd, IAA & KU500892 & 1,076 \\
\hline WP 4-10-4 & Curtobacterium sp. & Poplar leaf & Bright yellow & NA, IAA & KU550577 & 859 \\
\hline WP40 & Burkholderia sp. & Poplar stem & Buff & $\begin{array}{l}\text { ofn, orb, hcn, Sd, } \\
\text { NA, IAA }\end{array}$ & KF597274 & 1,494 \\
\hline WP41 & Burkholderia sp. & Poplar stem & Buff & $\begin{array}{l}\text { ofn, orb, Sd, NA, } \\
\text { IAA }\end{array}$ & KF597275 & 1,494 \\
\hline PD1 & Pseudomonas putida & Poplar stem & Buff & IAA & KF443801 & 1,496 \\
\hline Pf 2-79 & P. fluorescens & Soil & Bright yellow & CLP, phz & & \\
\hline Pf Q8r1-96 & P. brassicacearum & Soil & Buff & CLP, dapg, plt, prn & & \\
\hline
\end{tabular}

aPlant growth promoting activities: cyclic lipopetide (CLP) determined using the drop collapse assay; indole acetic acid (IAA) determined on YEM medium plus L-trpytophan using Salkowski reagent; gene clusters for the antifungal metabolites occidiofungin (ofn), ornibactin (orb), hydrogen cyanide (hcn), diacetylphloroglucinol (dapg), phenazine (phz), pyoluteorin (plt) and pyrrolnitrin (prn) loci determined using PCR; siderophore (Sd) determined by orange halo on CAS medium, nitrogenase activity (NA) determined by acetylene reduction assay. 